Michat Styczyński

Adam Mickiewicz University in Poznań,

Pedagogy and Fine Arts in Kalisz, Poland

\title{
II bisogno dei valori e ricerca del senso della vita dei giovani di oggi. Punti Chiavi della Logoterapia di Viktor E. Frankl applicati nel campo formativo
}

\section{Youth's need for values and search for the meaning of life. Key issues of the theory of logotherapy in the context of educational activities}

\begin{abstract}
During their existence, people often forget about the meaning of life. With the world spinning faster and faster, the disappearing ability to reflect on complex day-today life, the virtual reality and 'the net' jungle, people are forgetting how to discover values that lead to the higher goal. There is special concern for the young generation which often loses the meaning of life. The answer to the question - how to find the meaning of life - gives the theory of logotherapy and existential analysis, which was created by Victor E. Frankl. The story of his life, and the difficult experiences which led him to discover the meaning of life persuade the reader about the truthfulness of his scientific discoveries. Logotherapy is a way to find goals and meaning in life: it makes people think about their existence. Frankl shows a certain hierarchy of values, which appear during specific and often difficult life situations. He survived four concentration camps and during those extreme times he was able to find meaning in life. What is more, he helped others find their meaning. A teacher appears on a young man's life path and this teacher, according to logotherapy, should become the logo-educator and witness to the values. He should be someone, who points to meaning and is able to manifest the meaning of his own existence. Through a mature relationship with the student, the teacher helps the student discover what is the most important. The teacher helps the student realise one's own plans through to the will, meaning and human conscience - spaces through which the sens consciousness shines through.
\end{abstract}




\section{Keywords}

Logotherapy; existence; goal; meaning; value; logo-educator.

Ogni giorno l'uomo sperimenta una molteplicità di situazioni, relazioni ed impulsi che costituiscono il fondamento per le motivazioni delle concrete decisioni intraprese nel corso della propria vita. È ovvio che i processi decisivi sono indispensabili per poter realizzare in pieno la propria esistenza. Però, ultimamente si evidenzia purtroppo una mancanza di autostima e sicurezza di sé e l'incapacità di decidere in un modo responsabile. La realtà della vita propone tantissimi modi di autorealizzazione che perdono il loro significato. Uno dei ricercatori italiani descrive i nostri tempi con una frase abbastanza significativa, cioè l'importanza dell'inutile ${ }^{1}$. Ci si può domandare sul significato dell'inutile che attrae talmente l'uomo di oggi. L'autore appena citato cerca di dare risposta a quel problema presentando la propria opinione: «Avverto subito una difficoltà nell'uso di questo termine perché, paradossalmente, l'inutile è diventato essenziale, e io non accetterò mai di considerare necessario alla vita e alla sua qualità qualcosa che ha insito in sé il rischio di trasformarsi in danno» ${ }^{2}$.

L'inutile evoca il nichilismo che nega valori e significati delle cose, delle relazioni e delle azioni, e così, l'uomo non riesce a trovare il senso dell'esistenza. E' il problema che incontrano prima di tutto i giovani immersi nella molteplicità delle cose, relazioni e azioni alla cui mente è estranea una seria ed attenta riflessione sull'argomento cruciale della vita espresso nella domanda Che senso ha...?

\section{Logoterapia - risposta alla domanda sul senso}

L'uomo chiamato alla vita nella società e cultura cosidetta postmoderna è angosciato da un vuoto esistenziale «che corrisponde al senso di assurdità dell'esperienza, alla stanchezza di vivere, all'assenza di energia e di progettualità,

\footnotetext{
1 Cfr. V. Andreoli, L'educazione (im)possibile. Orientarsi in una società senza padri, Rizzoli, Milano 2014, p. 74.

2 V. Andreoli, L'educazione (im)possibile. Orientarsi in una società senza padri, p. 74.
} 
al crollo della voglia di lottare $\rangle^{3}$. La cultura postmoderna non offre quasi mai le possibilità di sperimentare qualcosa di veramente reale. Tutto è momentaneo e frammentato, privo di senso. In questa situazione «al giorno di oggi un numero sempre maggiore di individui dispone di risorse per vivere, ma non di un significato per cui vivere» ${ }^{4}$. L'osservazione proposta da Viktor E. Frank1 ${ }^{5}$, fondatore della logoterapia e dell'analisi esistenziale, mostra il grande impegno che quel personaggio eccezionale ha intrapreso per cercare e risanare l'uomo nella sua esistenza priva di significato.

Lo stesso Frankl, ebreo osservante e praticante, ha testimoniato con propria vita come rispondere alla domanda sul senso dell'esistenza. Quando al III Reich di Hitler fu annessa anche Austria la situazione di Frankl, in quanto ebreo, iniziò a complicarsi. Senza la possibilità di ottenere un visto per l'espatrio, decise di accettare la direzione del reparto di neurologia del Rothschildspital, con la speranza di essere salvato dall'internamento nei campi di concentramento. Ad un certo momento decise di sabotare il programma di eutanasia organizzato per i malati psichici dalle autorità tedesche attraverso la compilazione delle false diagnosi, salvando in questo modo anche i pazienti ebrei. Infine, ricevette il visto per l'espatrio. «Quando ottenne il visto per gli Stati Uniti, Frankl dovette porsi un problema di coscienza: avrebbe lasciato i suoi genitori da soli a fronteggiare il loro destino? Soltanto un ,segno dal cielo” lo fece desistere dall'intenzione di espatriare $\iota^{6}$. E così accadde. Una volta, a casa sua vide sul tavolo un frammento di marmo che suo padre trovò tra le rovine della sinagoga, distrutta dai tedeschi. Su questo pezzo ritrovò le prime parole di uno dei Comandamenti: Onora il padre e la madre affinché si prolunghino i tuoi giorni sulla terra. Così decise di restare con i suoi cari. In questo tempo sposò

3 L. Verdone, Emergenza educativa in un mondo che cambia, Edizioni Paoline, Milano 2009, p. 28.

4 V.E. Frankl, Un significato per l'esistenza. Psicoterapia e umanismo, Città Nuova Editrice, Roma 1990, p. 73-74.

5 Viktor Emil Frank1 (26.III.1905-2.IX.1997) medico psichiatra viennese, di origine ebrea. Egli fu incuriosito dalle opere di alcuni filosofi naturalisti, frequentò con piacere le lezioni di psicologia applicata e sperimentale (Freud, Adler, Allers, Schwarz). Nel 1926 per la prima volta durante una delle conferenze da lui tenute viene enunciata la parola e presentata la concezione della logoterapia, mentre l'analisi esistenziale, l'espressione alternativa, risale al 1933. Sulla base della propria teoria Frankl fondò i Centri di Consulenza giovanile con lo scopo di aiutare le persone che nella loro disperazione volevano suicidarsi. Nel periodo della II guerra mondiale riuscì a sopravvivere a quattro lager nazisti.

6 D. Bruzzone, Viktor Frankl. Fondamenti psicopedagogici dell'analisi esistenziale, Carocci Editore, Roma 2012, p. 30. 
Tilly Grosser, una donna di ingegno naturale e di delicatezza di sentimenti. Dopo nove mesi la famiglia intera fu deportata nel lager di Theresienstadt, e successivamente furono trasferiti ad Auschwitz. Prorpio ad Auschwitz prese la quintessenza del suo pensiero e di alcuni casi clinici ai quali applicò la sua teoria. Per fortuna una copia del manoscritto era stata consegnata da Frankl ad un suo amico e così si salvò. Nel campo di concentramento lo accompagnò la preghiera trovata nella tasca del suo nuovo vestito di campo, cioè la più importante orazione ebraica, lo Shemà Israel. Certamente, anche questa aiutò Frankl a rimanere in vita, nonostante la realtà eccessivamente atroce. Dovette sopportare il tifo petecchiale, la fame, il freddo, e seppe bravamente applicare la propria teoria proprio lì, nelle difficili o perfino "disumane" condizioni dei lager. Attraverso l'oggettivazione del dolore, prendendo distanza dal mondo circostante con la capacità umana di autotrascendenza, da lui stesso inventata, riuscì a sopravvivere a quattro lager: Theresienstadt, Auschwitz, Kaufering III e Türkheim. Perse il padre, la madre, il fratello e la moglie ${ }^{7}$. Da questa storia piena di dolore e carica delle situazioni insopportabili dal punto di vista umano nasce una nuova chiamata alla vita piena di senso, aperta ai valori incrollabili.

Dalla testimoninaza di Frankl che è riuscito ad applicare la sua teoria e nello stesso tempo la sua pratica terapeutica a sé stesso e agli altri nelle situazioni senza senso nasce una speranza anche per i giovani - figli della cultura postmoderna caratterizzata per la provvisorietà, accidentalità, imprevedibilità, esuberanza e caoticità, omonimia, asistematicità e irrazionalità. I sociologi contemporanei sottolineano che l'uomo è in una continua ricerca della sua identità, del senso della propria vita, ma non lo può ritrovare perché sperimenta una mancanza basilare - una mancanza dei principi di carattere universale ${ }^{8}$. Questa mancanza determina la crisi dell'uomo contemporaneo il quale privo di solidi fondamenti della sua esistenza, rimane senza la capacità di ritrovare il senso della propria vita. Bisogna, dunque, intraprendere uno sforzo, concreto e decisivo, per aiutare i giovani in ricerca del vero significato della loro vita conducendoli da un certo homunculus che reagisce agli stimoli oppure sfoga

7 Cfr. E. Fizzotti, Prefazione, in: V. E. Frankl, Lettere di un sopravvissuto. Ciò che mi ha salvato dal lager, Rubbettino Editore, Soveria Mannelli 2008, p. 5-10.

8 Cfr. M. Gałaś, Wartości kulturalne w epoce ponowoczesnej a wyzwania edukacyjne, in: J. Żebrowski (Ed.), Edukacja kulturalna w społeczeństwie obywatelskim, Gdańskie Towarzystwo Naukowe, Gdańsk 1997, p. 123. 
i propri istinti all'homo humanus che sa agire nel mondo'. Il potenziale umano si trova in ogni persona ma deve essere riscoperto. Non è vero che gli uomini umani sono in minoranza ${ }^{10}$. Anche se si deve affermare che nella vita dell'uomo di oggi c'è tanto negativismo, tanta violenza e solitudine, non dimentichiamo che nella profondità di ogni essere umano «c'è anche l'aspirazione, il bisogno a vivere pienamente la vita, in tutti i suoi aspetti, il bisogno d'essere attori sociali sempre più attivi» ${ }^{11}$.

\section{Logoterapia - scenario per i giovani di oggi}

L'educazione per diventare capace di formare bene l'uomo del XXI secolo, cioè per aiutarlo a conseguire una preparazione adeguata a un lavoro soddisfacente, a sviluppare creatività e partecipazione alla cultura, dovrebbe essere segnata da valori veri ${ }^{12}$. Sono infatti essi ad aprire nuove possibilità all'uomo per una vita piena di senso, specialmente ai giovani in ricerca del vero senso della vita. Una vita priva di senso li conduce inevitabilmente ai comportamenti che minacciano la loro esistenza. In tal modo «la logoterapia [...] gode di buona salute non solo perché le sue tecniche di intervento psicoterapeutico dimostrano ogni giorno più di avere efficacia e validità, ma anche perché offre continue sollecitazioni in chiave preventiva a quanti hanno a cuore lo sviluppo e la crescita della persona, e dei giovani in modo particolare. Sempre maggiore, infatti, è il numero di genitori, insegnanti, educatori, operatori sociali che chiedono di essere formati alla scuola della logoterapia perché ritengono che essa sia in grado di offrire una visione positiva dell'esistenza umana grazie alla quale favorire la ricerca di senso in un contesto socioculturale, quale quello contemporaneo, segnato inesorabilmente dal vuoto e dalla delusione» ${ }^{13}$.

9 Cfr. V.E. Frank1, Si può insegnare e imparare la psicoterapia? Scritti sulla logoterapia e analisi esistenziale, Edizioni Magi, Roma 2009, p. 43.

${ }^{10} \mathrm{Cfr}$. M. Wolicki, Podstawowe zagadnienia analizy egzystencjalnej i logoterapia, Wydawnictwo Diecezjalne, Sandomierz 2008, p. 57.

${ }^{11}$ M. Callari Galli, Il valore della differenza, in: P. Bertolini - M. Dallari (Edd.), Pedagogia al limite, La Nuova Italia, Scandicci (FI) 1991, p. 70.

${ }^{12}$ Cfr. E. Kobylecka, Wartości i cele wychowania, in: E. Kozioł-E. Kobylecka(Edd.), Pedagogika wobec problemów wychowania końca XX wieku, Zielona Góra 2000, WSP TK, p. 25.

${ }^{13}$ E. Fizzotti, Presentazione, in: V.E. Frankl, Ciò che non è scritto nei miei libri. Appunti autobiografici sulla vita come compito, Franco Angeli, Milano 2012, p. 8-9. 


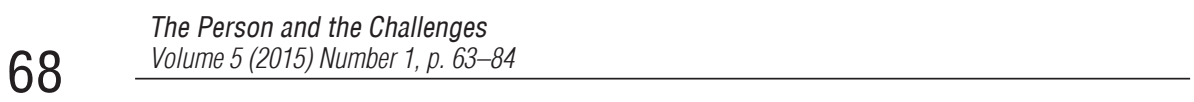

Il termine la vita senza significato diventa una caratteristica dellasituazione esistenziale dell'uomo di oggi. In tale contesto aiutano molto le visioni del mondo che, nelle proprie filosofie, tornano al vero senso dell'esistenza e ai veri valori ormai dimenticati. Specialmente oggi si sperimenta il vero bisogno della riscoperta del senso che, naturalmente, esiste ma cerca di essere ritrovato. Purtroppo le visioni del mondo, che sono in prevalenza concentrate su tutto questo e si presentano in un momento qui ed ora, spesso impediscono il ritrovamento del senso dell'esistenza umana. Per i nostri tempi, dunque, sembra essere una proposta valida e necessaria, l'educazione che tornasse all'essenziale, al senso, ai valori. La formazione dei giovani basata sui fondamenti valoriali, facilita tutto lo sforzo educativo e dovrebbe essere sempre centrata sul senso delle cose, delle relazioni, degli avvenimenti, ma prima di tutto dell'agire umano. L'analisi Esistenziale e la Logoterapia, come teoria che sa decifrare certi condizionamenti e certe caratteristiche della vita nella società dei nostri tempi, possono essere trattate come una proposta sussidiaria e molto efficace per tutti gli ambienti e livelli delle azioni formative di oggi.

\section{Dalla volontà di significato al senso della vita}

Il nucleo centrale nella teoria frankliana è la volontà di significato, che costituisce la motivazione e il bisogno fondamentale dell'uomo. A tale problematica egli si è interessato fin dall'adolescenza quando, volendo diventare medico, si fermava spesso a riflettere sulle questioni filosofiche relative alla vita e alla morte, come pure sul significato ultimo della vita umana ${ }^{14}$. Egli, maturando meglio le sue intuizioni giovanili, è giunto ad affermare che la realizzazione dell'esistenza può effettuarsi solamente attraverso la realizzazione di un significato che però è collocato sempre al di fuori di essa ${ }^{15}$. La stessa parola esistenza (dal latino ex-sistere) significa appunto «uscire da se stesso e confrontarsi con se stesso» ${ }^{16}$. In questo modo, come osserva Marshall riferendosi al pensiero frankliano,

${ }^{14}$ Cfr. M. Marshall, Life with Meaning. Guide to the Fundamental Principles of Viktor E. Frankl's Logotherapy, Maritime Institute of Logotherapy, Halifax, Nova Scotia, Canada 2009, p. 6.

${ }^{15}$ Cfr. V.E. Frankl, Fondamenti e applicazioni della logoterapia, Società Editrice Internazionale, Torino 1977, p. 47.

${ }_{16}$ P. Giovetti, Viktor Frankl. Vita e opere del fondatore della logoterapia, Edizioni Mediterranee, Roma 2001, p. 122. 
l'esistenza non è un puro fatto d'essere, ma un facoltativo modo di essere, un percorso attraverso il quale l'uomo diventa veramente se stesso ${ }^{17}$.

L'uomo essendo in possesso di questa specifica caratteristica è stato 'equipaggiato' per trovare il vero senso dell'esistenza che lo rende veramente felice. Incontrando il significato della sua vita, l'uomo ritrova la vera possibilità dello sviluppo, del divenire, della realizzazione di sé, dell'essere personale. L'esistenza è un'esperienza molto profonda e personale che si compie non solo attraverso i processi biopsichici, ma anche tramite l'attività propria del soggetto, delle sue scelte, delle sue aspirazioni e decisioni ${ }^{18}$.

Ogni persona aperta alla ricerca di senso riesce a trascendere se stesso, grazie alla qualità specificamente umana denominata da Frankl volontà di significato che definisce «la tensione radicale dell'uomo a trovare e realizzare un senso e uno scopo» ${ }^{19}$. L'uomo, in altri termini, cerca continuamente un senso da dare alla propria esistenza e ciò risveglia in lui un'innata volontà di significato che si realizza mediante un'analisi fenomenologica dell'esperienza immediata e autentica ${ }^{20}$.

Secondo l'impostazione teorica della logoterapia frankliana il significato assume un ruolo importante e fondamentale nella vita di ogni persona. Dal punto di vista oggettivo il significato si concentra sul senso dell'esistenza. Infatti, non esiste la vita umana prima di senso, non ci sono le situazioni senza senso. C'è, dunque, il bisogno e nello stesso tempo il compito di scoprire il detto significato. Così come la vita umana è unica in se stessa anche il significato è unico e concreto. A volte l'uomo deve sperimentare qualcosa che sembra troppo difficile da sopportare. Per questo bisogna imparare una specifica abilità che garantisce il raggiungimento del senso esistenziale chiamata forza di resistenza. Di solito le situazioni diventano, con questa forza, più ricche di significato ${ }^{21}$. Il significato possiede anche il carattere soggettivo. Prima di tutto è ascritto a una persona concreta che cerca il senso della propria vita in una determinata

\footnotetext{
${ }^{17}$ Cfr. M. Marshall, Life with Meaning, p. 16.

${ }^{18}$ Cfr. K. Popielski, Noetyczny wymiar osobowości. Psychologiczna analiza poczucia sensu życia, Redakcja Wydawnictw KUL, Lublin 1994, p. 23.

${ }_{19}$ V. E. Frank1, La sfida del significato. Analisi esistenziale e ricerca di senso, Erickson, Trento 2005, p. 59.

${ }^{20}$ Cfr. R. Zavalloni, Psicologia della speranza. Per sentirsi realizzati, Edizioni Paoline, Milano 1994, p. 175.

${ }^{21}$ Cfr. M. Styczyński, Il bisogno del significato nella vita dell'uomo secondo la visione logoterapeutica di Victor E. Frankl, „Teologia i człowiek” (2012) 20, p. 182-184.
} 
situazione nella quale si scopre il vero senso. L'altra caratteristica importante del significato dal punto di vista soggettivistico consiste nell'impossibilità di inventarlo. Il significato è sempre riferito ad personam e ad situationem ${ }^{22}$. E' notevole che „il senso dell'esistenza esige il senso dell'essere, che talvolta Frankl chiama «senso ultimo» o «sovrasignificato»" 23 . L'uomo trascendendosi e superando se stesso infine si avvicina ad un senso totale e universale. Il fattore che aiuta l'uomo nella ricerca di senso e rafforza le possibilità umane è incontestabilmente la fede religiosa ${ }^{24}$.

\section{Verso il senso attraverso i valori}

Una lettura realistica della cultura del nostro tempo fa emergere con chiarezza come l'uomo abbia perso tutto ciò che possa avere valore e sia degno di vivere, che gli dia senso d'identità. In apparenza, l'uomo ha tutto quello che può dargli „benessere”, ma in realtà gli manca qualcosa che è difficile da descrivere, ma che nello stesso momento è fondamentale. Perché l'uomo possa trovare una risposta circa la natura del suo essere e il significato del suo "malessere" ha bisogno di una più esplicita e completa visione antropologica che consideri fondamentale la ricerca di significati e di valori. L'analisi esistenziale frankliana nella sua specificità si riferisce prima di tutto all'orientamento dell'uomo verso il senso come pure all'aspirazione verso $i$ valori ${ }^{25}$. In ambedue le direttrici si vede una grande preoccupazione per lo sviluppo di ogni persona umana, per la dignità dell'uomo. Ogni persona si trova nel processo del continuo sviluppo: „persona” si diventa e non è giusto affermare Io sono come sono ${ }^{26}$. In questo incessante sviluppo personale l'uomo è guidato da un sistema di valori. Nella vita quotidiana occorre riconoscere la loro gerarchia, poiché «avere un insieme di valori non gerarchizzati non giustificherebbe un orientamento valoriale della persona in un senso piuttosto che in un altro» ${ }^{27}$. In ogni situazione esistenziale

${ }^{22}$ Cfr. M. Styczyński, Il bisogno del significato, p. 185.

23 D. Bruzzone, Viktor Frankl. Fondamenti psicopedagogici, p. 113.

${ }^{24}$ Cfr. M. Styczyński, Il bisogno del significato, p. 186-187.

${ }^{25}$ Cfr. V.E. Frank1, Homo patiens. Soffrire con dignità, Queriniana, Brescia 2001, p. 25.

${ }^{26}$ Cfr. V.E. Frankl, Homo patiens, p. 63.

${ }^{27}$ D. Bellantoni, Le prospettive cliniche della logoterapia. Verso la definizione di un modello clinico integrato, in: Fizzotti E. (Ed.), Nuovi orizzonti di ben-essere esistenziale. Il contributo della logoterapia di V.E. Frankl, LAS, Roma 2005, p. 163. 
un adeguato sistema di valori garantisce lo sviluppo e la maturazione della personalità, aiuta il processo dell'integrazione come pure determina certe qualità verso le quali si rivolgono le attività di una persona ${ }^{28}$. Il percorso di vita che si affida ai valori potrebbe «dischiudere all'uomo la vera gioia» ${ }^{29}$.

La visione dei valori di Frankl fa chiaro riferimento al pensiero di Max Scheler. La logoterapia di Frankl e l'etica dei valori di Scheler trovano un punto comune prima di tutto nel riconoscimento dell'esistenza spirituale dell'essere umano ${ }^{30}$. Il filosofo tedesco sostiene che la persona umana capace di trascendenza diventa centro di atti intenzionali e di valori. L'uomo, cioè, è capace di «comportarsi come un essere illimitatamente aperto al mondo. Diventar uomini significa elevarsi, in forza dello spirito, fino a potersi aprire al mondo ${ }^{31}$. Anche Frankl mette in evidenza che la persona umana è capace di autotrascendenza verso un mondo di valori e di significati. L'uomo, in quanto essere-nel-mondo, si indirizza verso uno scopo, un significato da cercare e verso i valori da realizzare ${ }^{32}$. Entrambi sono d'accordo che «i valori sono e restano infatti essenze di un universo trascendente la coscienza che li intende. (...) I valori esistono quindi secondo un ordine gerarchico indipendente dal giudizio della singola persona che li percepisce, anzi, normativo per qualsiasi giudizio di tipo etico $»^{33}$.

Si deve notare che la presenza di un ordine gerarchico dei valori risolverebbe il problema della collisione dei valori ${ }^{34}$. Secondo Frankl «l'impressione che due valori siano in collisione tra loro è conseguenza del fatto che viene trascurata un'intera dimensione. E qual è questa dimensione? È l'ordine gerarchico dei valori. Secondo Max Scheler, la valutazione implica il preferire un valore ad un altro. Questo è il risultato finale della sua profonda analisi fenomenologica dei processi di valutazione. Il grado di un valore è sperimentato insieme al valore

${ }^{28}$ Cfr. K. Popielski, Noetyczny wymiar osobowości, p. 127.

${ }^{29}$ V. E. Frankl, Logoterapia e analisi esistenziale, Morcelliana, Brescia 2005, p. 76.

${ }^{30}$ Cfr. E. Fizzotti, Logoterapia per tutti, Guida teorico-pratica per chi cerca il senso della vita, Rubbettino, Soveria Mannelli 2002, p. 75.

${ }^{31}$ M. Scheler, La posizione dell'uomo nel cosmo e altri saggi, Milano 1970, Fabbri, p. 183.

${ }^{32}$ Cfr. V.E. Frank1, Dio nell'inconscio. Psicoterapia e religione, Morcelliana, Brescia 1975, p. $39-40$.

${ }_{33}$ D. Bruzzone, Autotrascendenza e formazione. Esperienza esistenziale, prospettive pedagogiche e sollecitazioni educative nel pensiero di Viktor E. Frankl, Vita e Pensiero, Milano 2001, p. 115.

${ }^{34}$ Cfr. V.E. Frankl, Fondamenti e applicazioni della logoterapia, p. 65. 
stesso. In altri termini, l'esperienza di un valore implica l'esperienza che esso si colloca più in alto di un altro. Non c'è posto per conflitti di valore» ${ }^{35}$.

Si tratta di cogliere la percezione del valore nel suo significato universale. In un'epoca segnata dal crollo delle tradizioni, e di conseguenza dalla svalutazione dei valori, sembra sia cresciuto il sentimento della mancanza di senso. Pur assegnando al valore un significato universale, Frankl sostiene che «non esiste un significato universale della vita ma (...) esistono significati unici di situazioni individuali. Tuttavia non dobbiamo dimenticare che fra queste situazioni vi sono anche di quelle che hanno qualcosa in comune e, conseguentemente, vi sono anche i significati condivisi da esseri umani attraverso la società e, ancor più, attraverso la storia. Piuttosto che essere in relazione a situazioni uniche, tali significati hanno riferimento alla condizione umana. Sono questi significati che noi chiamiamo valori. Cosicché si possono definire $i$ valori come quei significati universali che cristallizzano delle situazioni tipiche che la società o l'umanità intera deve affrontare $\rangle^{36}$.

Se l'uomo nella sua essenza cerca la propria realizzazione, essa può compiersi solamente attraverso la realizzazione dei valori in quanto significati universali che sono a-temporali e sovratemporali ${ }^{37}$. In quest'ottica emerge con chiarezza la dimensione temporale, cioè il fatto che l'assunzione del valore, la norma di comportamento è verificata nel tempo. Ogni uomo deve essere cosciente che il valore, in quanto tale, aspetta sempre di essere realizzato. Riferendosi ai "valori situazionali" di Scheler, Frank1 così li definisce: «sono valori che, per così dire, attendono la loro ora per venire realizzati, il momento in cui un uomo afferra l'occasione unica per attuarli; se si fallisce l'occasione, essa è irrimediabile perduta e il valore situazionale rimane per sempre irrealizzato; l'uomo l'ha frustrato»" ${ }^{38}$.

Il secondo elemento che caratterizza la concezione valoriale di Frankl è lo sforzo per cogliere i nuovi valori nell'esistenza umana. Ogni situazione esistenziale si presenta all'uomo come una specie di domanda esistenziale che lo chiama a un compito specifico. L'uomo sia dunque l'essere che «invece di interrogarsi di continuo sul senso della vita, si sentisse chiamato a rispondere,

${ }^{35}$ V. E. Frank1, Senso e valori per l'esistenza. La risposta della Logoterapia, Nuova Editrice, Roma 1998, p. 70.

${ }^{36}$ V. E. Frankl, Fondamenti e applicazioni della logoterapia, p. 64.

${ }^{37}$ Cfr. V.E. Frankl, Le radici della logoterapia. Scritti giovanili 1923-1942, LAS, Roma 2000, p. 117.

${ }^{38}$ V. E. Frank1, Logoterapia e analisi esistenziale, p. 93. 
si sentisse come uno a cui la vita pone sempre delle domande, come un essere collocato in mezzo ad un'infinità di compiti. La psicologia insegna che "cogliere il senso" sta ad un livello più elevato del "dare senso" $"$ ". E ciò si può eseguire con la presenza attiva della coscienza umana - il vero organo di senso ${ }^{40}$.

L'idea di una triplice tipologia di valore secondo Frankl attraverso cui l'uomo può cogliere il significato della vita nasce nel 1929. Così egli scrive nei suoi appunti autobiografici: «Queste tre possibilità di dare un senso alla vita sono:

a) un'azione che l'uomo compie o un'opera che realizza;

b) un'esperienza, un incontro o un atto d'amore;

c) ma anche quando ci troviamo di fronte ad un destino ineluttabile (pensiamo a una malattia inguaribile, un carcinoma inoperabile), anche in questa situazione possiamo strappare un senso alla vita, dando testimonianza della più umana fra le capacità umane: quella di trasfigurare la sofferenza in una prestazione umana $\rangle^{41}$.

Si tratta, in altri termini, dei valori di creazione, valori di esperienza e valori di atteggiamento. La prima categoria dei valori si riferisce a tutto quello che l'uomo è capace di dare al mondo con la propria capacità creativa. I valori di esperienza invece accentuano tutto quello che l'uomo prende dal mondo, tutto ciò che l'uomo accoglie: la bellezza, la verità come pure la persona dell'altro. La terza categoria prende in considerazione l'atteggiamento che l'uomo assume confrontandosi con tutte le situazioni esistenziali, in quanto ineluttabili e inevitabili ${ }^{42}$.

Questa proposta valoriale offre un'indicazione importante per cercare i veri valori che sono le ragioni dell'esistenza umana vissuta in un modo consapevole, libero e responsabile.

\section{L'educatore come testimone dei valori}

Un educatore che opera nel contesto odierno, dovrebbe «possedere in sé - ben prima e ben oltre la qualifica professionale, le competenze disciplinari

${ }^{39}$ V. E. Frankl, Le radici della logoterapia, p. 119-120.

${ }^{40}$ Cfr. D. Bellantoni, Le prospettive cliniche della logoterapia, p. 164.

${ }^{41}$ V. E. Frankl, La vita come compito: appunti autobiografici, Torino 1997, Società Editrice Internazionale, p. 42.

${ }^{42}$ M. E. Viscardi, Esistenza ed etica secondo Viktor E. Frankl. Per una rifondazione della prassi terapeutica, "Ricerca di Senso" 6 (2008) 1, p. 65-66. 
e le relative abilità didattiche - le qualità umane e le virtù che si ritiene di dover promuovere nell'educando ${ }^{43}$. In ambito educativo, infatti, ciò che conta è l'essere più che il fare o il dire. È la stessa personalità dell'educatore che è direttamente coinvolta nell'azione educativa.

Occorre recuperare nella situazione educativa attuale, come un importante fattore del processo educativo, la dimensione della intenzionalità, che oggi purtroppo si sta perdendo. Invece, sono proprio le interazioni educative 'intenzionali', che influenzano la formazione della personalità. Esse sono caratterizzate da una 'colorazione assiologica e morale', e proprio come tali, rendono possibile la maturazione dell'uomo. L'uomo non è uno qualsiasi ma diventa il qualcuno che conta. Per attraversare questo passaggio è necessario che l'azione educativa sia dinamica e di qualità ${ }^{44}$.

La logoterapia frankliana ha la capacità di ridonare a ogni interazione educativa sia una dinamica sia una qualità speciale. Ciò vuol dire che «la ricerca di senso si traduce in un movimento intenzionale verso i significati e i valori, che rappresenta l'autentico dinamismo formativo che l'educazione dovrebbe promuovere» ${ }^{45}$.

L'agire educativo implica sempre una certa assiologia, infatti, ogni intervento dell'educatore viene 'decodificato' dall'educando, nel quadro di un determinato orizzonte valoriale ${ }^{46}$. La presenza personale nella relazione educativa rimane in qualche modo influenzata dalla cultura postmoderna, che a volte dimentica la dignità della persona e il bisogno di una vita significativa, concentrandosi sull'aspetto puramente tecnico e metodico dell'uomo, rischiando di spegnere quello spirituale. L'oggettivizzazione dell'uomo, operata dalle scienze umane, non rende conto di un valore primario che è proprio la persona ${ }^{47}$. Purtroppo sia i giovani sia gli educatori trascurano l'importanza della dimensione interiore e così fanno fatica a costruire la propria esistenza.

43 D. Bruzzone, Autotrascendenza e formazione, p. 360.

${ }^{44}$ Cfr. K. Ablewicz, Człowiek jako metodologiczny problem pedagogiki, in: W. Pasierbek, M. Grodecka (Eds.), Horyzonty wychowania w czasach przemian, WAM, Kraków 2009, p. 543.

45 D. Bruzzone, L'educazione al senso e il senso dell'educazione. Implicazioni rivoluzionarie di un'intuizione pedagogica, "Ricerca di Senso" 4 (2006) 1, p. 28.

${ }^{46}$ Cfr. D. Bruzzone, L'educazione al senso, p. 544.

${ }^{47}$ Cfr. K. Jasińska-S. Ruciński, Istota i formy patologii moralnej oraz jej społeczne uwarunkowania, in: F. Adamski (Ed.), Wartości - Społeczeństwo - Wychowanie. Studia z pedagogiki społecznej, Wyd. UJ, Kraków 1995, p. 22-23. 
Dal punto di vista evolutivo, verso i 18 anni - come emerge dalle ricerche psicologiche - la persona entra nello stadio di sviluppo segnato dal bisogno di una autenticità delle regole e dei valori. Questo periodo a volte coincide con un certo fanatismo del giovane che insiste sulle proprie opinioni e opzioni valoriali senza considerare la presenza dell'altro con le proprie preferenze esistenziali ${ }^{48}$. In questo caso è importante individuare una modalità efficace per poter educare i giovani alla negoziazione e discussione, come pure al confronto e all'accordo con gli altri ${ }^{49}$.

I giovani di oggi, immersi nella cultura postmoderna, non hanno purtroppo ben chiare prospettive quali siano le regole e valori per loro importanti. Manca, in realtà, il materiale su cui discutere, manca la scelta concreta per certi valori da parte dei giovani. La secolarizzazione dei valori morali, come pure l'individualizzazione, è all'origine della mancanza di identificazione con qualcosa di significativo. Il relativismo morale che cancella dall'orizzonte assiologico i valori spirituali, e negando Dio conduce alla negazione dell'uomo stesso ${ }^{50}$. Questi sintomi che, descrivono la situazione attuale, contribuiscono altresì alla negazione e cancellazione dell'autorità e delle norme ${ }^{51}$. Se poi si aggiunge la mancanza di educatori 'autorevoli' e consapevoli della propria vocazione, ci troviamo in una situazione che è priva di stabili fondamenti nel processo educativo $^{52}$. In realtà, mentre i giovani stanno cercando delle figure significative nel loro processo di costruzione dell'identità, «la figura dell'insegnante sembra essere in crisi e ciò finisce per entrare in cortocircuito con la ricerca d'identità, sempre più confusa e sofferta, da parte degli allievi. In una simile situazione, gli insegnanti sono tentati di chiudersi in se stessi e di limitarsi al solo ruolo didattico, rinunciando al ruolo molto più impegnativo e coinvolgente qual è quello educativo. Due ruoli, questi, che, se si punta a un insegnamento e a un apprendimento efficaci, devono invece essere strettamente legati tra loro» ${ }^{53}$.

${ }^{48}$ Cfr. A. Błasiak, Aksjologiczne aspekty procesu wychowania. Wybrane zagadnienia, WAM, Kraków 2009, p. 115.

${ }^{49}$ Cfr. A. Błasiak, Aksjologiczne aspekty procesu wychowania, p. 115-116.

${ }^{50}$ Cfr. K. Chałas, Wychowanie ku wartościom. Elementy teorii i praktyki. Tom I, Jedność, LublinKielce 2006, p. 96-97.

${ }^{51}$ Cfr. K. Chałas, Wychowanie ku wartościom, p. 97.

${ }^{52}$ Cfr. K. Chałas, Wychowanie ku wartościom, p. 21.

${ }^{53}$ F. Pergola, L'inconscio a scuola. Psicodinamica dei processi d'insegnamento e apprendimento, in: F. Pergola (Ed.), L'insegnante sufficientemente buono. Psicodinamica della relazione educativa docente - allievo - scuola, Magi, Roma 2010, p. 19. 
I giovani hanno bisogno di educatori che stiano loro vicini, non solo nei momenti più difficili della loro crescita, ma anche come persone che sanno testimoniare un'esistenza piena di senso e fondata sui valori consistenti. La formazione è collegata in qualche modo al principio di imitazione. L'individuo sottoposto al processo educativo ha bisogno di qualcuno da imitare ${ }^{54}$. Però il principio di imitazione viene sempre associato alla valorizzazione del mondo circostante. Si potrebbe dire che la realtà è di carattere assiologico, cioè contiene certi valori esistenziali che si devono scoprire. Attraverso la realizzazione degli eventi esistenziali si formano i valori e proprio in questo processo l'educatore deve accompagnare sempre la vera formazione ${ }^{55}$. Per formare una persona matura e responsabile, bisogna concentrarsi sugli obiettivi-valori educativi che costituiscono l'ideale dell'educazione. Nel processo educativo questo ideale deve essere incarnato in una concreta esemplarità personale da parte di qualcuno che, annunciando certi valori, sa testimoniare la possibilità di realizzarli nella propria esistenza ${ }^{56}$.

Il principio dell'imitazione di un modello identificativo è in pieno accordo con la proposta frankliana. In tempi di insicurezza formativa, causata da tanti fattori del tempo postmoderno, la figura del logo-educatore, che mediante la sua professione testimonia i valori fondanti dell'esistenza, diventa un supporto importante per gli educatori di oggi in ricerca del modello educativo efficace. «Secondo un principio metodologico classico, che Frankl ripetutamente richiama (...) l'educazione non si dà se non all'interno di un rapporto interpersonale, asimmetrico e tuttavia non autoritario, in cui l'educatore, più che limitarsi a trasmettere una supposta verità assoluta, ha il compito di testimoniarne esemplarmente la significatività sul piano esistenziale e personale» ${ }^{57}$.

L'importanza della testimonianza nell'agire educativo assume un ruolo prioritario, se si pensa che «il nichilismo dei giovani è rinforzato (se non addirittura

${ }^{54}$ Cfr. K. Wrońska, Aksjologiczne podstawy zasady naśladownictwa w wychowaniu, in: F. Adamski (Ed.), Wartości - Społeczeństwo - Wychowanie. Studia z pedagogiki społecznej, Wyd. UJ, Kraków 1995, p. 103.

${ }^{55}$ Cfr. J.H. Estrada, Educación para el corazón «El hombre en busca de sentido», "LOGO: Teoría, Terapia, Actitud" 11 (1995) 20, p. 16.

${ }^{56}$ Cfr. K. Wrońska, Aksjologiczne podstawy zasady naśladownictwa, p. 103-104.

${ }^{57}$ D. Bruzzone, Autotrascendenza e formazione, p. 348. 
provocato) dal cinismo degli adulti, dalla carenza di modelli significativi e di valori che non siano proclamati in forma retorica, ma autenticamente vissuti $\rangle^{58}$.

A questo punto bisogna ricordare uno dei postulati frankliani, secondo il quale bisogna educare secondo un paradigma anti-omeostatico della tension reduction. Si tratta, cioè, di dare ai giovani la possibilità di vivere in una certa tensione esistenziale che offrirebbe l'occasione per incarnare il vero senso della vita attraverso i valori concreti e vissuti ${ }^{59}$. L'educazione, cioè, deve tener conto di questa tensione tra la persona e lo scopo che intende raggiungere, un compito, un traguardo, un esempio da imitare, un valore da attuare.

Per poter svolgere il proprio ruolo in un modo efficace, l'educatore dovrà essere per il giovane una persona credibile ${ }^{60}$. Presentandosi egli stesso per primo come un vero testimone dei valori e comportandosi in coerenza con le proprie preferenze valoriali, il logo-educatore diventa l'esempio che orienta l'educando nelle sue scelte valoriali, nel processo di gerarchizzazione dei valori come pure nella risoluzione delle situazioni esistenziali in riferimento a dei valori concreti che potrebbero essere realizzati ${ }^{61}$.

Possiamo dire che oltre alla credibilità dell'educatore, è di fondamentale importanza la sua autenticità. L'educatore, testimoniando le proprie convinzioni, accende nel giovane il bisogno di un'esistenza significativa fondata su sicure basi valoriali. Attraverso le situazioni esistenziali con le quali si confronta l'educatore, egli stesso diventa un vero testimone per i giovani che lo osservano ${ }^{62}$. Sono proprio queste situazioni che necessitano una chiara opzione valoriale. L'educatore competente, in senso logoterapeutico, è sempre pronto a vivere secondo certi valori con i quali si risponde alle concrete situazioni della vita quotidiana. In questo modo è possibile offrire un cospicuo sostegno ai giovani alla ricerca del senso e dei valori ${ }^{63}$.

${ }^{58}$ D. Bruzzone, Dal logo-terapeuta al logo-educatore. Perché la logoterapia non è soltanto terapia, "Ricerca di Senso" 6 (2008) 3, p. 319-320.

${ }^{59}$ Cfr. P.B. Etchebehere, Apuntes para una Logo - Pedagogía, "LOGO: Teoria, Terapia, Actitud" 8 (1992) 14, p. 12-13.

${ }^{60}$ Cfr. D. Bruzzone, L'educazione al senso e il senso dell'educazione, p. 36.

${ }^{61}$ Cfr. K. Biller, Sinnzentriert Erziehen! - Darstellung und Begründung, "Existenz und Logos. Zeitschrift für sinnzentrierte Therapie Beratung Bildung" 13 (2005) 2, p. 14.

${ }^{62}$ Cfr. K. Biller, Sinnzentriert Erziehen!, p. 14.

${ }^{63}$ Cfr. M. Wolicki, Logoterapeutyczna koncepcja wychowania, Wydawnictwo Diecezjalne, Sandomierz 2007, p. 156. 
L'educatore-testimone, inoltre, dovrebbe possedere una caratteristica essenziale tipica di ogni processo formativo: la capacità di motivare. L'educazione ai valori comporta prima di tutto il fare appello alla libertà responsabile del giovane, orientandolo verso la realizzazione dei valori trascendenti. Il giovane, se è ben motivato, riesce a superare tutti i condizionamenti biologici, psicologici e sociali grazie alla chiara visione valoriale che gli è stata proposta ${ }^{64}$. Il logoeducatore nell'accompagnare i giovani li aiuta innanzitutto a compiere delle scelte valoriali e ad accogliere valori che garantiscono il raggiungimento del significato della loro esistenza. In seguito, li aiuterà a scoprire i nuovi valori, in coerenza con le preferenze già assunte ${ }^{65}$.

Verso quali valori orientare oggi il giovane? L'educatore, testimone dei valori attraverso il proprio comportamento, le scelte e la propria personalità, attesta la sua visione esistenziale. Egli fa da "mediatore", cioè da via privilegiata per favorire nell'educando l'incontro con i valori, e ciò è possibile nella misura in cui l'educatore per primo ha una personalità sostanzialmente aperta ai valori e al senso della vita. In questo consiste la sua testimonianza educativa, nel proporsi, cioè, egli stesso come persona alla ricerca dei valori che danno significato alla vita.

Il giovane riesce a decifrare dal comportamento dell'educatore le sue preferenze valoriali e anche riferendosi al suo esempio, comincia a creare i nuovi valori. Però, la capacità di scegliere certi valori e abbandonare gli altri si fonda sulla libertà individuale, in riferimento all'obiettivo concreto, senza cadere nell'oggettivismo. Quando si parla del senso e dei valori è utile assumere un quadro di valori obiettivi oppure universali.

Parlando di proposta valoriale, è lecito richiamare la visione frankliana dei valori presentata già precedentemente. Frankl distingue tre categorie di valori: valori di creazione, valori di esperienza e valori di atteggiamento, che si possono accostare ai trascendentalia di Platone. Si potrebbe affermare con F. Larocca che «l'intuizione dei Valori supremi fin da bambini agisce potentemente su ogni azione produttiva. L'amore dei Valori supremi o di qualcuno di essi, stimola ad esercitare e preparare le funzioni fisio-psichiche più necessarie. Ma soprattutto è la facoltà di desiderio, quella di realizzare la felicità, che, una volta che abbia intuito ed amato i Valori supremi, li persegue con indefessa fedeltà, costringendo

${ }^{64}$ Cfr. N.B. Boado de Landaboure, La relación pedagógica como encuentro existencial, "LOGO: Teoria, Terapia, Actitud" 13 (1997) 24, p. 22.

${ }^{65}$ Cfr. K. Biller, Sinnzentriert Erziehen! - Darstellung und Begründung, p. 14. 
tutte le altre funzioni ad organizzarsi e piegarsi per realizzare qualche anticipo di Verità - Bene - Bello - Uno» ${ }^{66}$.

Leggendo in questa chiave i valori di Frankl, si può offrire grande supporto per la funzione educativa del logo-educatore. La testimonianza di vita dei valori di creazione, di esperienza e di atteggiamento, che richiamano in un certo modo tutto ciò che Bello, Buono e Vero, può trovare la sua applicazione nella vita dei giovani, favorendo processi di unificazione personale, attraverso la costruzione della propria identità. Solo quando si coniugano il Buono, il Bello e il Vero si risveglia l'anima dell'educando e nello stesso tempo esso si apre al vero senso della vita ${ }^{67}$.

Cominciando dai valori creativi, si potrebbe paragonarli con il Bonum dell'uomo. Il Bonum è addirittura ciò che è prezioso, di valore. Il Bonum è una categoria semplice che non si riesce a smembrare. Non è possibile definirlo per genus et differentium perché è summum genus di tutta la gamma delle qualificazioni ${ }^{68}$. Anche oggi esiste il summum bonum che Aristotele ha definito come eudaimonia ( $\varepsilon v \delta \alpha \iota \mu v \imath \alpha$ ). Essa è legata con l'agire degno della persona, l'agire ragionevole e virtuoso, e proprio così non può essere inteso come astrazione, ma come una realizzazione dell'uomo ${ }^{69}$.

Nonostante tutto, si deve ricordare che l'uomo non riuscirà a trovare i veri valori chiudendosi in se stesso, bensì aprendosi alla dimensione trascendentale di fronte a lui. Solo così ogni individuo può crescere e diventare una persona matura $^{70}$. In questo modo è possibile il raggiungimento del senso attraverso la realizzazione dei valori creativi.

La seconda categoria dei valori frankliani ruota attorno all'esperienza della persona. Tutto questo segnala un'altra capacità specificamente umana: la capacità di godimento ${ }^{71}$. È possibile realizzare i valori anche «nell'esperienza della vita, nel modo con cui si accolgono i doni che il mondo ci offre, nel modo con cui accogliamo ammirati la bellezza della natura o dell'arte $\rangle^{72}$. È lecito, parlando dei valori di esperienza, rievocare l'esperienza artistica, filosofica, letteraria, ma

\footnotetext{
${ }^{66}$ F. Larocca, Oltre la creatività: l'educazione, La Scuola, Brescia 1983, p. 124.

${ }^{67}$ Cfr. P.B. Etchebehere, Apuntes para una Logo - Pedagogía, p. 12.

${ }^{68}$ Cfr. W. Tatarkiewicz, O filozofii i sztuce, PWN, Warszawa 1986, p. 79-80.

${ }^{69}$ Cfr. S. Michałowski, Pedagogia wartości, Wyd. Debit, Bielsko Biała 1993, p. 36.

${ }^{70}$ Cfr. K. Denek, Ku dobrej edukacji, Wyd. Akapit, Toruń - Leszno 2005, p. 132-133.

${ }^{71}$ Cfr. V.E. Frankl, Logoterapia medicina dell'anima, Gribaudi Editore, Milano 2001, p. 124.

72 V. E. Frank1, Logoterapia e analisi esistenziale, p. 84.
} 
anche quella classica - amorosa ${ }^{73}$. Il nostro autore è del parere che il significato può essere trovato «sperimentando qualcosa o qualcuno, e fare esperienza di qualcuno nella sua unicità e irripetibilità vuol dire amarlo» ${ }^{74}$.

Esaminando i trascendentalia di Platone si coglie come la categoria del Bello trovi corrispondenza con i valori dell'esperienza di Frankl. Infatti, tutto quello che è Bello fa parte dell'esperienza umana. «Si tratta di formare un atteggiamento profondo ed universale dell'uomo verso il Bello, di trasferire l'arte dai musei nella vita, garantendole una degna accoglienza. (...) Il Bello non può perdersi nelle piccolezze dei profitti e della propaganda, deve essere un'esperienza personale profonda e sconvolgente» ${ }^{75}$.

Ogni uomo possiede la capacità di percepire la realtà. Già Platone notava che le cose belle sono oggetto della percezione, non nozioni della realtà. L'oggetto delle nozioni invece è il Bello stesso ${ }^{76}$. Aristotele, con la sua filosofia di mezzo cominciava guardare la realtà del Bello con l'influsso dei fattori formali e, specialmente nell'ambiente dell'arte, dei fattori affettivi ${ }^{77}$.

L'ultima classe dei valori nominati da Frankl è data dai valori di atteggiamento. La concezione logoterapeutica vede nella realizzazione dei valori di atteggiamento la prestazione più alta ${ }^{78}$. Essa proviene dal fatto che «quando dobbiamo affrontare un destino che semplicemente non si può cambiare $\mathrm{o}$, meglio, che al momento non si può cambiare (...) ebbene, anche quando ci troviamo come vittime indifese in una situazione senza speranza, anche là, anzi proprio là, la vita può essere piena di senso: perché allora possiamo realizzare ciò che di più umano vi è nell'uomo, e al contempo testimoniare la più umana di tutte le capacità umane: quella di trasformare una tragedia in un trionfo personale, una sofferenza in una prestazione umana» ${ }^{79}$.

Da questo punto di vista i valori di atteggiamento si mostrano come i più significativi. Certamente, la dimensione del significato della sofferenza non

${ }^{73}$ Cfr. E. Fizzotti, Logoterapia per tutti, p. 160.

${ }^{74}$ V. E. Frankl, Si può insegnare e imparare la psicoterpai? Scritti sulla logoterapia e analisi esistenziale, p. 57.

${ }^{75}$ P. Lombardo, Educare ai valori. società - famiglia. Compiti e ruoli educativi, Vita Nuova, Verona 2003, p. 270.

${ }^{76}$ Cfr. W. Tatarkiewicz, Historia filozofii. Tom pierwszy, PWN, Warszawa 1983, p. 87.

${ }^{77}$ Cfr. W. Tatarkiewicz, Historia filozofii, p. 119.

${ }^{78}$ Cfr. V.E. Frankl, Logoterapia medicina dell'anima, p. 124.

${ }^{79}$ V. E. Frankl, Si può insegnare e imparare la psicoterapia? Scritti sulla logoterapia e analisi esistenziale, p. 57. 
è la stessa dei valori creativi oppure di esperienza ${ }^{80}$. La realizzazione del valore di atteggiamento non si riferisce solo al dolore di origine biologica; di essa si parla anche nella realizzazione di un valore di carattere noetico ${ }^{81}$. Si deve infine notare, che «i valori si pongono davanti a noi come domande, come appello: suscitano, chiedono risposte. $\mathrm{Ci}$ sono persone che hanno sperimentato in questo modo che nel dolore c'è la possibilità concreta di crescere, che si è realizzato ciò che prima appariva non possibile» ${ }^{82}$.

Anche se le possibilità umane sembrano essere scarse, nel caso di dover affrontare una sfida difficile, l'uomo deve comportarsi in un modo degno dischiudendo il nuovo regno di valori che garantiscono la propria realizzazione personale ${ }^{83}$.

Una caratteristica molto vicina ai valori di atteggiamento è la concezione della Verità di Platone. Questa categoria, comune e basilare in ogni azione umana, viene associata con la domanda fondamentale che tutti i ricercatori si pongono: Che cos'è la Verità? Secondo Aristotele, che attraverso la Verità voleva esprimere la compatibilità fra idea e realtà, essa è nella mente, nelle operazioni mentali, nel giudizio, e solo così può spettare a tutto ${ }^{84}$. Nel nostro tempo «la Verità è divenuta ciò che è remunerativo e redditizio, comodo e ambito. Ciò che contraddistingue il nostro tempo è il culto della tecnica e dei miti che si accompagna ad un relativismo storico e sociale. Ma non potrà durare a lungo: si deve rinnovare la capacità di vivere un profondo senso della verità sovra individuale» ${ }^{85}$. In tal senso, giungendo alla Verità, si possono realizzare, in modo adeguato, i valori di atteggiamento.

La riflessione appena presentata ci dà alcune indicazioni che potrebbero aiutare il logo-educatore nella sua funzione di testimone dei valori. Il percorso esistenziale dell'educatore-accompagnatore dell'educando in ricerca apre un cammino molto significativo, dove essi «si trovano, in tal modo, rivolti

${ }^{80}$ Cfr. V.E. Frankl, La sofferenza di una vita senza senso. Psicoterapia per l'uomo di oggi, Elledici, Torino 1978, p. 83.

${ }^{81}$ Cfr. A. Gismondi, Autodistanziamento e autotrascendenza. Risorse umane: in ogni condizione, in: E. Fizzotti (Ed.), Il senso come terapia. Fondamenti teorico-clinici della logoterapia di Victor E. Frankl, Franco Angeli, Milano 2007, p. 119.

${ }^{82}$ I. Punzi, Significato e valore della sofferenza, in: E. Fizzotti (Ed.), Il senso come terapia. Fondamenti teorico-clinici della logoterapia di Victor E. Frankl, Franco Angeli, Milano 2007, p. 130.

${ }^{83}$ Cfr. V. E. Frankl, Logoterapia e analisi esistenziale, p. 85.

${ }^{84}$ Cfr. J. Legowicz, Filozofia. Istnienie Myślenie Działanie, PWN, Warszawa 1972, p. 173-174.

${ }^{85}$ P. Lombardo, Educare ai valori, p. 269. 
contemporaneamente verso l'individuazione di valori che vanno considerati non come delle mete già raggiunte, ma come orizzonti esistenziali verso i quali tendere sempre e con rinnovato impegno» ${ }^{86}$.

Secondo l'impostazione frankliana «l'educatore non rinuncia al proprio ruolo di portatore-testimone di valori, ma soltanto ai riflessi coercitivi che esso può implicare ${ }^{87}$. Il processo educativo fondato nel rapporto personale tra educatore ed educando richiede, da parte del primo, un impegno notevole, una scienza profonda, una pazienza, ma prima di tutto un alto grado di responsabilità ${ }^{88}$. Ciò risulta possibile se al di là delle dichiarazioni di principio da parte dell'educatore c'è una testimonianza di vita vissuta in coerenza con i valori proclamati. In effetti, il logo-educatore deve essere «un testimone di come si sta al mondo, per aiutare gli altri a starci con senso» ${ }^{89}$. In tal modo il ruolo dell'educatore diventerà sempre più convincente e rivolto verso determinati scopi che garantiscono la buona riuscita formativa degli educandi.

\section{Conclusioni}

La ricerca di senso nella nostra società sembra essersi fatta più difficile. La sfida per ogni uomo, specialmente per un giovane, sta nel continuo sforzo per raggiungere la vera felicità. Non c'è l'altra strada per questo compito esistenziale che aprire e aprirsi a nuovi orizzonti di senso. Tutto ciò invoca la presenza di educatori che si rendano capaci di 'mediazione', che si trasformino, cioè, in 'facilitatori'. E questo comporta che in primo luogo siano essi stessi persone che abbiano cercato e trovato il senso della propria vita, che abbiamo, pur nella fatica e nella lotta, dato un senso al dolore, e si siano costruiti una personale gerarchia di valori. Da qui la necessità di docenti che siano prima di tutto 'educatori', meglio ancora, 'logo-educatori'. Allora il processo di ricerca di senso e l'educazione ai valori, affidati alla cura del logo-educatore, cioè di una persona che è capace di incontrare l'altro in profondità, mediante una relazione 'significativa', nello spirito di amore e di comprensione, saranno facilitati da un

${ }^{86}$ E. Fizzotti, Prefazione, in: D. Bruzzone, Autotrascendenza e formazione. Esperienza esistenziale, prospettive pedagogiche e sollecitazioni educative nel pensiero di Viktor E. Frankl, Vita e Pensiero, Milano 2001, p. XII.

${ }^{87}$ D. Bruzzone, Autotrascendenza e formazione, p. 354.

${ }^{88}$ Cfr. M. Wolicki, Logoterapeutyczna koncepcja wychowania, p. 156.

${ }^{89}$ D. Bruzzone, Dal logo-terapeuta al logo-educatore, p. 331. 
clima relazionale ricco di valori, un accompagnamento personale e reciproco, che apre insieme a nuovi orizzonti di valori, e in seguito, di senso.

\section{Bibliography}

Ablewicz K., Człowiek jako metodologiczny problem pedagogiki, in: W. Pasierbek, M. Grodecka (Eds.), Horyzonty wychowania w czasach przemian, WAM, Kraków 2009, p. 543-559.

Andreoli V., L'educazione (im)possibile. Orientarsi in una società senza padri, Rizzoli, Milano 2014.

Bellantoni D., Le prospettive cliniche della logoterapia. Verso la definizione di un modello clinico integrato, in: E. Fizzotti (Ed.), Nuovi orizzonti di ben-essere esistenziale. Il contributo della logoterapia di V.E. Frankl, LAS, Roma 2005, p. 147-171.

Błasiak A., Aksjologiczne aspekty procesu wychowania. Wybrane zagadnienia, WAM, Kraków 2009.

Bruzzone D., Autotrascendenza e formazione. Esperienza esistenziale, prospettive pedagogiche e sollecitazioni educative nel pensiero di Viktor E. Frankl, Vita e Pensiero, Milano 2001.

Bruzzone D., L'educazione al senso e il senso dell'educazione. Implicazioni rivoluzionarie di un'intuizione pedagogica, "Ricerca di Senso" 4 (2006) 1, p. 25-36.

Bruzzone D., Viktor Frankl. Fondamenti psicopedagogici dell'analisi esistenziale, Carocci Editore, Roma 2012.

Callari Galli M., Il valore della differenza, in: P. Bertolini, M. Dallari (Eds.), Pedagogia al limite, La Nuova Italia, Scandicci (FI) 1991, p. 69-78.

Chałas K., Wychowanie ku wartościom. Elementy teorii i praktyki. Tom I, Jedność, LublinKielce 2006.

Fizzotti E., Logoterapia per tutti, Guida teorico-pratica per chi cerca il senso della vita, Rubbettino, Soveria Mannelli 2002.

Fizzotti E., Prefazione, in: V.E. Frankl, Lettere di un sopravvissuto. Ciò che mi ha salvato dal lager, Rubbettino Editore, Soveria Mannelli 2008, p. 5-25.

Fizzotti E., Presentazione, in: V.E. Frankl, Ciò che non è scritto nei miei libri. Appunti autobiografici sulla vita come compito, Franco Angeli, Milano 2012, p. 7-11.

Frankl V.E., Dio nell'inconscio. Psicoterapia e religione, Morcelliana, Brescia 1975.

Frankl V.E., Fondamenti e applicazioni della logoterapia, Società Editrice Internazionale, Torino 1977.

Frankl V.E., Homo patiens. Soffrire con dignità, Queriniana, Brescia 2001.

Frank1 V.E., La sfida del significato. Analisi esistenziale e ricerca di senso, Erickson, Trento 2005.

Frankl V.E., La vita come compito: appunti autobiografici, Torino, Società Editrice Internazionale, 1997.

Frankl V.E., Le radici della logoterapia. Scritti giovanili 1923-1942, LAS, Roma 2000.

Frankl V.E., Logoterapia e analisi esistenziale, Morcelliana, Brescia 2005.

Frankl V.E., Senso e valori per l'esistenza. La risposta della Logoterapia, Nuova Editrice, Roma 1998. 
Frankl V.E., Si può insegnare e imparare la psicoterapia? Scritti sulla logoterapia e analisi esistenziale, Edizioni Magi, Roma 2009.

Frankl V.E., Un significato per l'esistenza. Psicoterapia e umanismo, Città Nuova Editrice, Roma 1990.

Gałaś M., Wartości kulturalne w epoce ponowoczesnej a wyzwania edukacyjne, in: J. Żebrowski (Ed.), Edukacja kulturalna w społeczeństwie obywatelskim, Gdańskie Towarzystwo Naukowe, Gdańsk 1997, p. 117-129.

Giovetti P., Viktor Frankl. Vita e opere del fondatore della logoterapia, Edizioni Mediterranee, Roma 2001.

Jasińska K. - Ruciński S., Istota i formy patologii moralnej oraz jej spoteczne uwarunkowania, in: F. Adamski (Ed.), Wartości - Społeczeństwo - Wychowanie. Studia z pedagogiki społecznej, Wyd. UJ, Kraków 1995, p. 21-31.

Kobylecka E., Wartości i cele wychowania, in: E. Kozioł, E. Kobylecka (Eds.), Pedagogika wobec problemów wychowania końca XX wieku, Zielona Góra, WSP TK, 2000, p. 21-27.

Marshall M., Life with Meaning. Guide to the Fundamental Principles of Viktor E. Frankl's Logotherapy, Maritime Institute of Logotherapy, Halifax, Nova Scotia, Canada 2009.

Popielski K., Noetyczny wymiar osobowości. Psychologiczna analiza poczucia sensu życia, Redakcja Wydawnictw KUL, Lublin 1994.

Scheler M., La posizione dell'uomo nel cosmo e altri saggi, Milano, Fabbri 1970.

Styczyński M., Il bisogno del significato nella vita dell'uomo secondo la visione logoterapeutica di Victor E. Frankl „Teologia i człowiek” (2012) 20, p. 173-192.

Verdone L., Emergenza educativa in un mondo che cambia, Edizioni Paoline, Milano 2009.

Viscardi M.E., Esistenza ed etica secondo Viktor E. Frankl. Per una rifondazione della prassi terapeutica, "Ricerca di Senso" 6 (2008) 1, p. 57-72.

Wolicki M., Podstawowe zagadnienia analizy egzystencjalnej i logoterapia, Wydawnictwo Diecezjalne, Sandomierz 2008.

Zavalloni R., Psicologia della speranza. Per sentirsi realizzati, Edizioni Paoline, Milano 1994. 